\title{
Safety of Cerebrolysin for neurorecovery after acute ischemic stroke: a systematic review and meta-analysis of twelve ran- domized-controlled trials
}

\author{
Stefan Strilciuc ${ }^{1}$, László Vécsei ${ }^{2}$, Dana Boering ${ }^{3}$, Aleš Pražnikar ${ }^{4}$, Peter Riederer 5,6, Leontino Battistin 7 \\ 1 Department of Neuroscience, Iuliu Hatieganu University of Medicine and Pharmacy, Romania \\ 2 Department of Neurology, University of Szeged, Hungary \\ 3 SRH Gesundheitszentrum Bad Wimpfen, Bad Wimpfen, Germany \\ 4 Institute for Neurological Sciences, Queen Elisabeth University Hospital, Glasgow, Ireland \\ 5 Clinic and Policlinic for Psychiatry, Psychosomatics and Psychotherapy, University Hospital Wuerzburg, \\ Wuerzburg, Germany \\ 6 Department of Psychiatry, University Southern Denmark, Odense, Denmark \\ 7 Department of Neurosciences, University of Padova, Italy \\ * Correspondence: strilciuc.stefan@umfcluj.ro.
}

\begin{abstract}
We performed a systematic search and meta-analysis of available literature to determine the safety profile of Cerebrolysin in acute ischemic stroke, filling existing safety information gaps and inconsistent results. We searched EMBASE (Excerpta Medica Database, 1947 to March 2021), MEDLINE (1946 to March 2021), CENTRAL (1948 to March 2021) and Cochrane Database of Systematic Reviews (1995 to March 2021). Data collection and analysis was conducted using methods described in the Cochrane Handbook for Systematic Reviews of Interventions. All safety outcomes were analyzed based on risk ratios (RR) and their 95\% confidence intervals. The meta-analysis pooled 2202 patients from twelve randomized clinical trials, registering non-statistically significant ( $p>0.05)$ differences between Cerebrolysin and placebo throughout main and subgroup analyses. The lowest rate of Serious Adverse Events (SAE), as compared to placebo, was observed for the highest dose of Cerebrolysin $(50 \mathrm{~mL})$, highlighting a moderat reduction $(\mathrm{RR}=0.6)$. We observed a tendency of superiority of Cerebrolysin regarding SAE in high dose treatment courses for moderatesevere ischemic stroke, suggesting some effect of the agent against adverse events. This comprehensive safety meta-analysis confirms the safety profile for patients treated with Cerebrolysin after acute ischemic stroke, as compared to placebo.
\end{abstract}

Keywords: ischemic stroke; safety; cerebrolysin; neurorehabilitation

\section{Introduction}

Ischemic stroke continues to have overwhelming impact on health of populations and is expected to maintain its leading contribution to global mortality well into this century [1]. Studies have shown that post-stroke patients experience a wide range of adverse outcomes, such as aphasia, post-stroke anxiety, and depression, among others. Patientlevel health outcomes for acute ischemic stroke have significantly improved in the last decade primarily because of superior overall case management, availability of tailored drug interventions, and advances in endovascular procedures. Nevertheless, health systems face a "care gap" particularly due to the ongoing COVID-19 pandemic, as well as other factors that hamper provision of quality services [2]. Several factors, including financing and infrastructure constraints, limited expertise, and clinical uncertainty, still prevent adherence to evidence-based clinical guidelines and optimal care pathways [3].

Cerebrolysin is a combination of peptides that mimick the biological effect of neurotrophic factors, and amino acids obtained from highly purified lipid-free porcine brain proteins that promotes neurotrophic stimulation (survival and maintaining the 
phenotype of highly differentiated cells), neuroprotection against noxious agents, neuromodulation (e.g. changes in neuronal and synaptic plasticity), and metabolic regulation (i.e. against lactic acidosis and an increase in resilience against hypoxic conditions) [4]. Randomized clinical trials have highlighted the efficacy of cerebrolysin in motor and neurological function recovery following AIS [5,6].

Cerebrolysin is recommended in clinical practice guidelines across several continents [7-9]. Previous meta-analyses on Cerebrolysin safety profile provided inconsistent results. This applies especially the two largest most recent meta-analyses: Bornstein et al. 2018, including 1879 patients from nine randomized-controlled trials (RCTs) [10], and the review of Ziganshina et al. 2020, including 1601 patients from seven RCTs [11]. In order to resolve the reported discrepancies, the present meta-analysis aimed to explore the safety profile of Cerebrolysin, using the maximum amount of evidence available.

\section{Materials and Methods}

\subsection{Study selection and information sources}

This systematic review and meta-analysis included randomized, double-blind, placebo-controlled, clinical studies completed until February 28th, 2021, and assessing efficacy of Cerebrolysin as add-on treatment to standard care of ischemic stroke and published as full-text articles were considered as eligible for inclusion in this meta-analysis. No restrictions were placed on language, publication (year, type, or status), study endpoint (duration, length of follow-up, type of outcome measures) or treatment intervention (treatment window, dosage, frequency, duration). Studies that did not provide outcome data or data usable for the meta-analysis as well as studies that did not meet the inclusion criteria were excluded. Safety parameters were adverse events, serious adverse events, and death.

Information was sourced from Embase, PubMed and the Cochrane Database of Systematic Reviews up to end of February 2021. To further identify studies for this review, we also screened major review references and study registries (ClinicalTrials.gov, https://clinicaltrials.gov/; ISRCTN registry, http://www.isrctn.com/). We contacted authors of unpublished but registered studies and the producer of Cerebrolysin, to provide additional evidence and references for the meta-analysis. The search term "Cerebrolysin" was applied to all electronic database searches. The search strategy for Embase was ('cerebrolysin'/exp OR Cerebrolysin) and for PubMed it was ("cerebrolysin"[Supplementary Concept] OR "cerebrolysin"[All Fields]). No filters were used. Title, authors, and details of the periodical of the retrieved records have been listed on an Excel spreadsheet and screened by two independent researchers to remove identical records. The title and the abstract (when available) of the remaining records were scrutinized and obviously irrelevant reports have been excluded. We arranged for the complete reports of the remaining references and for professional translation services if published in languages other than English. After examination of the full text reports potentially relevant studies have been identified and all related records were promoted to the stage of data extraction. Studies identified in registries of completed or unknown status were scrutinized for eligibility and cross-checked with retrieved citation. The flow of the search process is presented in Figure 1. 


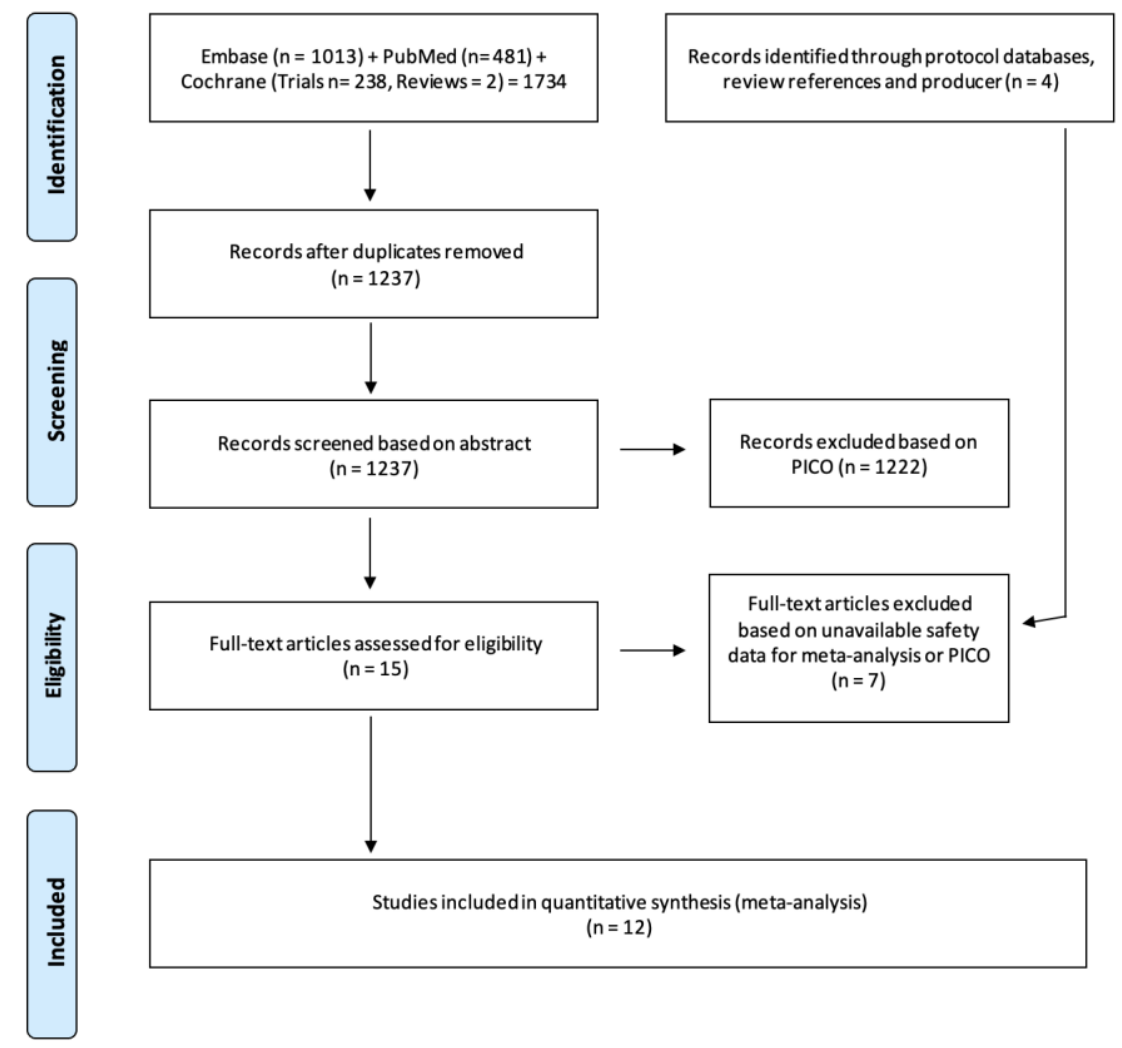

Figure 1. PRISMA diagram highlighting study selection process

If publications were not providing all details necessary for a comprehesive safety evluation, supplementary study documents were requested from the original authors (such as Study Protocols, Clinical Study Reports, etc.). Data from each included publication was extracted by two reviewers, working independently and using an extraction form which was devised for the study. Each included RCT was assessed for selection, performance, detection, attrition and reporting bias, and other bias that might have been detected during the review process. Disagreement regarding the extracted elements, classification of evidence, or assessment of effect size was resolved by consensus; if consensus was not obtained, a third team member was involved. Inclusion of any supplements for a specific trial was documented in the footnotes of the RoB table. In addition, individual patient data (IPD) were obtained for the following RCTs: Gharagozli et al. 2011, Heiss et al. 2012, Lang et al. 2012, Muresanu et al. 2016, and Guekht et al. 2015 [5,12-15]. Aggregate data from publication and individual patient data were cross-validated. In case of discrepancies the original authorswere contacted for clarification. All discrepancies could be resolved and were related to different underlying data sets (safety, ITT, FAS). For one trial no information on AE and SAE could be retrieved. This study was excluded from the corresponding analyses.

\subsection{Statistical analysis}

The safety outcomes were as follows: all-cause deaths, patients with at least one adverse event (AE), patients with at least one serious adverse event (SAE), and patients with at least one non-fatal serious adverse event (NFSAE). All safety outcomes were analyzed based on risk ratios (RR) and their $95 \%$ confidence intervals (CI). In one study no information was available on AE and SAE. This study was omitted from the corresponding analysis. We applied a random effects model (DerSimonian-Laird), based on the risk ratio (RR) as effect size for the binary safety criteria. Effect sizes were presented with $95 \%$ CIs and associated P-values. Heterogeneity was assessed by means of the I-squared (I2) 
procedure. All meta-analyses were performed using Revman (Version 5.4, The Cochrane Collaboration). In addition to the pooled analyses across all included randomized trials, sensitivity analyses were performed using the following stratification categories, including subsequent pooling across subgroups and formal tests for interaction:

- $\quad 20-30 \mathrm{~mL}$ vs. $50 \mathrm{~mL}$

- $20-30 \mathrm{~mL}<20$ Days vs. $20-30 \mathrm{~mL} \geq 20$ Days

- $50 \mathrm{~mL}<20$ Days vs. $50 \mathrm{~mL} \geq 20$ Days

- $\quad$ Treatment Initiation Within 24 Hours vs. Treatment Initiation > 24 Hours

- $\quad$ Studies published independently and available online.

For all subgroup analyses, tests for subgroup interaction and subgroup heterogeneity were performed based on Chi2 test and I2. A significance level of $\alpha=0.05$ was used a threshold for data interpretation. Risk of bias (RoB) assessment for the safety evaluations was performed using all available data from original publications. In unclear cases, supplementary study documents were requested from the original authors (such as Study Protocols, Clinical Study Reports, etc.). Inclusion of any supplements for a specific trial was documented in the footnotes of the RoB table.

\section{Results}

Twelve trials met the inclusion criteria, providing safety data for use of Cerebrolysin for 2202 from a total of 2274 randomized patients in the studies selected for formal analysis (Table 1).

Table 1. Description of studies and populations included in formal analyses

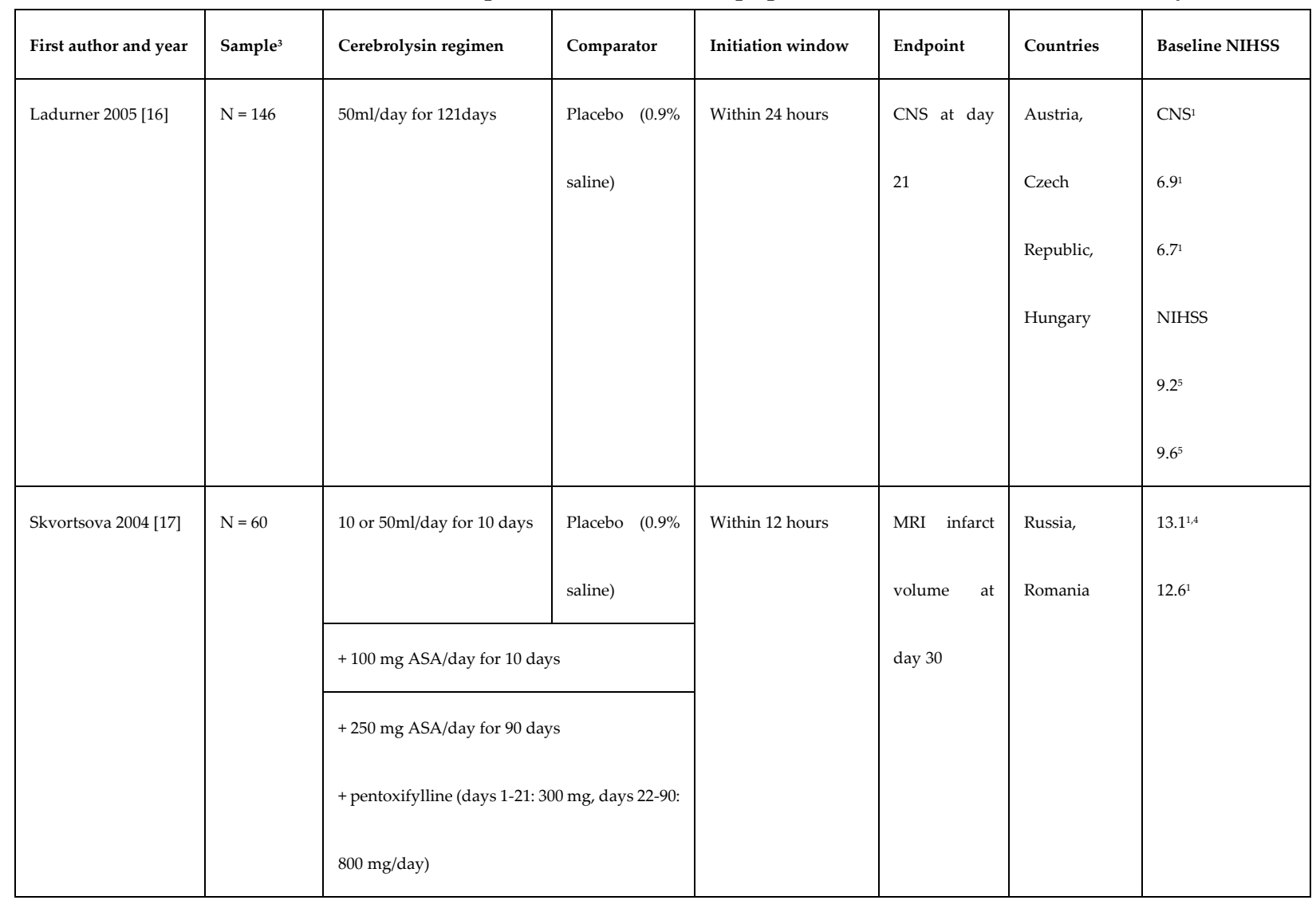




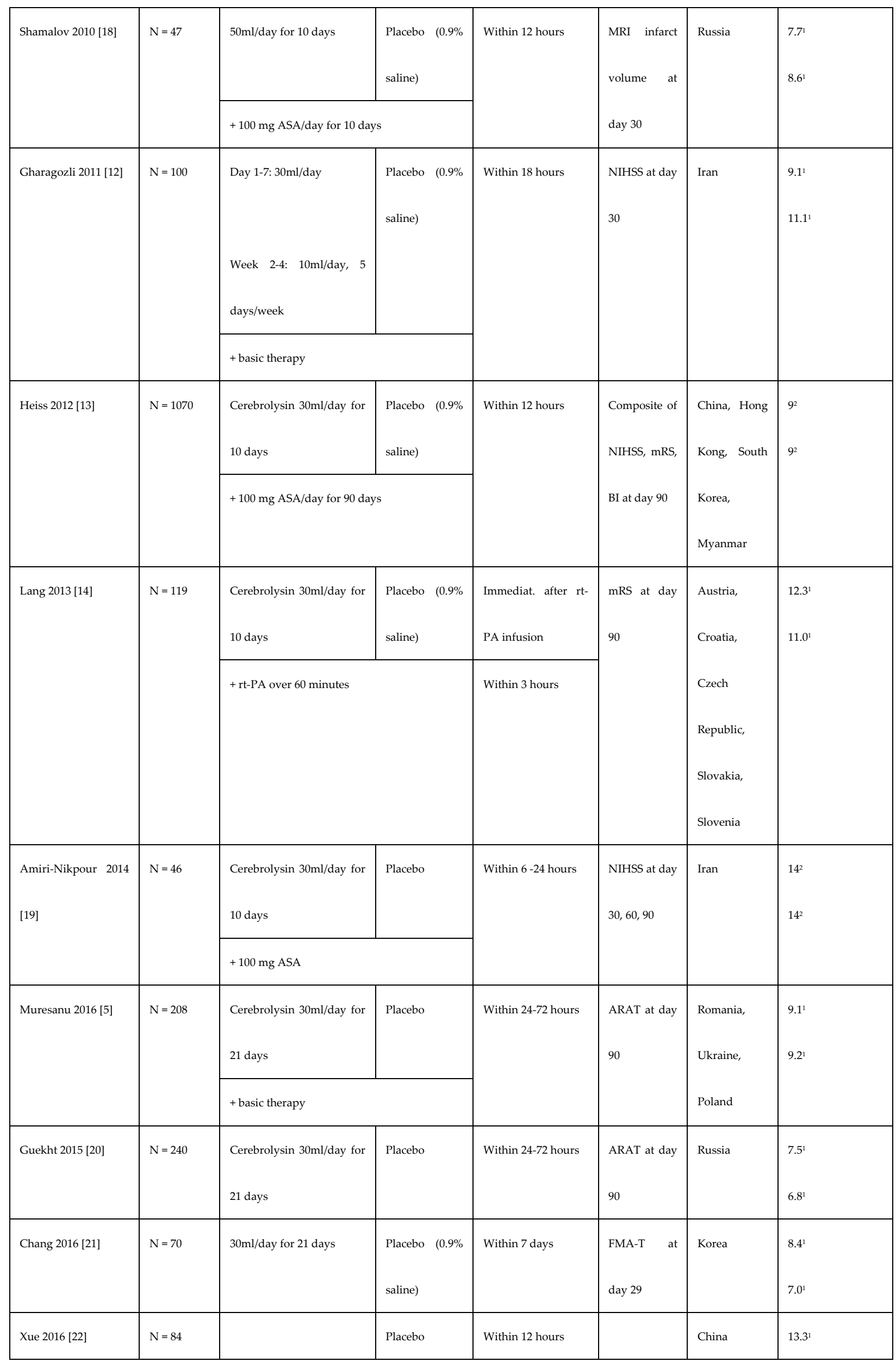




\begin{tabular}{|c|c|c|c|c|c|c|c|c|}
\hline & & $\begin{array}{l}\text { Cerebrolysin } 30 \mathrm{ml} / \mathrm{da} \\
10 \text { days }\end{array}$ & & NBP & & $\begin{array}{l}\text { NIHSS and BI } \\
\text { Day } 30\end{array}$ & & $12.7^{1}$ \\
\hline & & \multicolumn{3}{|l|}{ + basic therapy } & & Day 30 & & \\
\hline \multirow[t]{2}{*}{ Stan 2017 [6] } & $\mathrm{N}=84$ & Cerebrolysin & \multicolumn{2}{|c|}{ Placebo } & Within 48 hours & NIHSS & Romania & $8.9^{1}$ \\
\hline & & $30 \mathrm{ml} /$ day for 10 days & & & & Day 30 & & $7.8^{1}$ \\
\hline
\end{tabular}

${ }^{1}$ means (Cerebrolysin vs placebo),

${ }^{2}$ medians (Cerebrolysin vs placebo),

${ }^{3}$ all randomized groups,

$450 \mathrm{ml}$ group

${ }^{5}$ No NIHSS available, NIHSS derived from CNS using validated conversion model (Nilanont et al. The Canadian Neurological Scale and the NIHSS: Development and Validation of a Simple Conversion Model. Cerebrovasc Dis 2010;30;120-126.Doi: 10.1159/000314715)

\subsection{Deaths}

Crude pooling of deaths across studies resulted in a total of 45 deaths out of 1101 subjects treated with Cerebrolysin (4.1\%), as compared to 55 deaths out of 1101 subjects treated with placebo (5.0\%). Deaths were evaluated by means of the risk ratio (RR). The combined RR for deaths of all cause was resulting in a small superiority of Cerebrolysin with risk reduction of deaths by $17 \%$, which was statistically not significant with $\mathrm{P}=0.36$ $(\mathrm{RR}=0.83,95 \% \mathrm{CI}=0.57$ to $1.23, \mathrm{P}=0.36$, random effects model, Figure 2$)$.

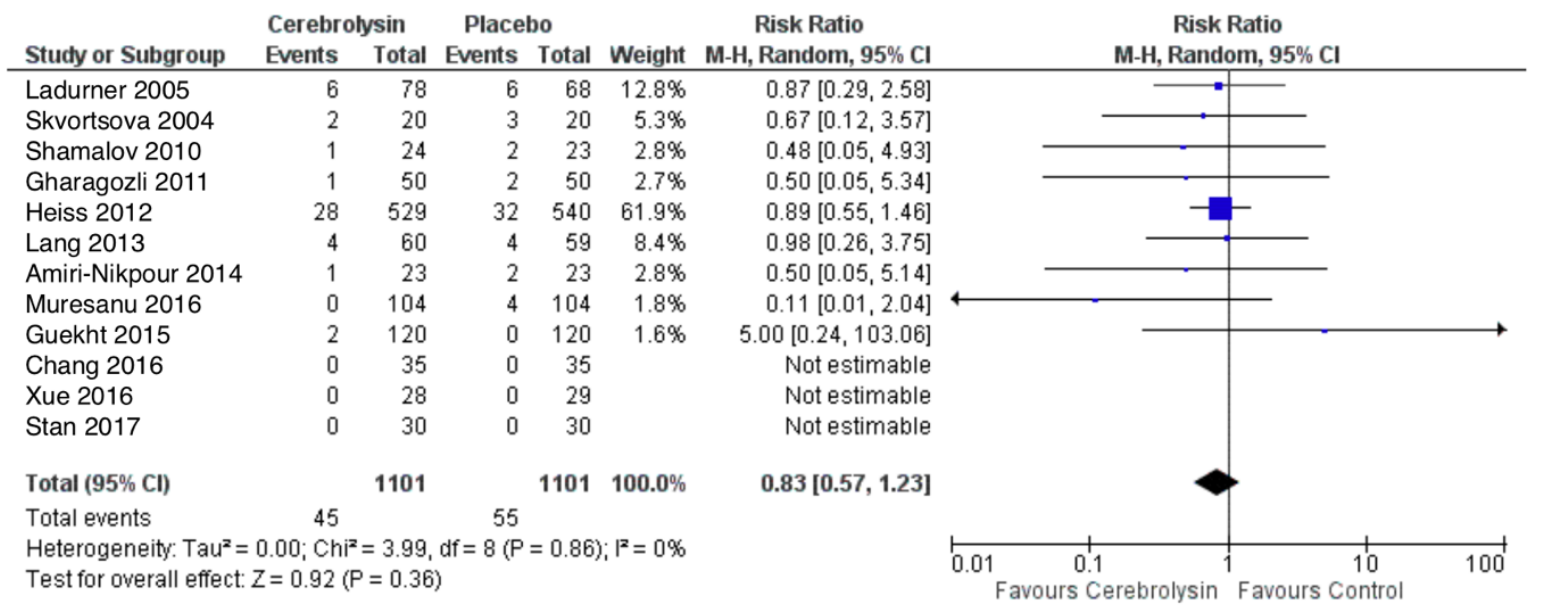

Figure 2. Deaths (All-cause); Comparison of Cerebrolysin versus Placebo, Safety Population, Random Effects, M-H, Risk Ratio (RR)

\subsection{Serious adverse events ( $S A E)$}

SAE were reported in a total of 85 out of 1078 subjects treated with Cerebrolysin (7.9\%), as compared to 85 out of 1076 subjects treated with placebo (7.9\%). The combined RR for patients with at least one SAE showed no difference between the groups $(\mathrm{RR}=0.99$, $95 \% \mathrm{CI}=0.74$ to $1.32, \mathrm{P}=0.95$, random effects model, Figure 3 ). 


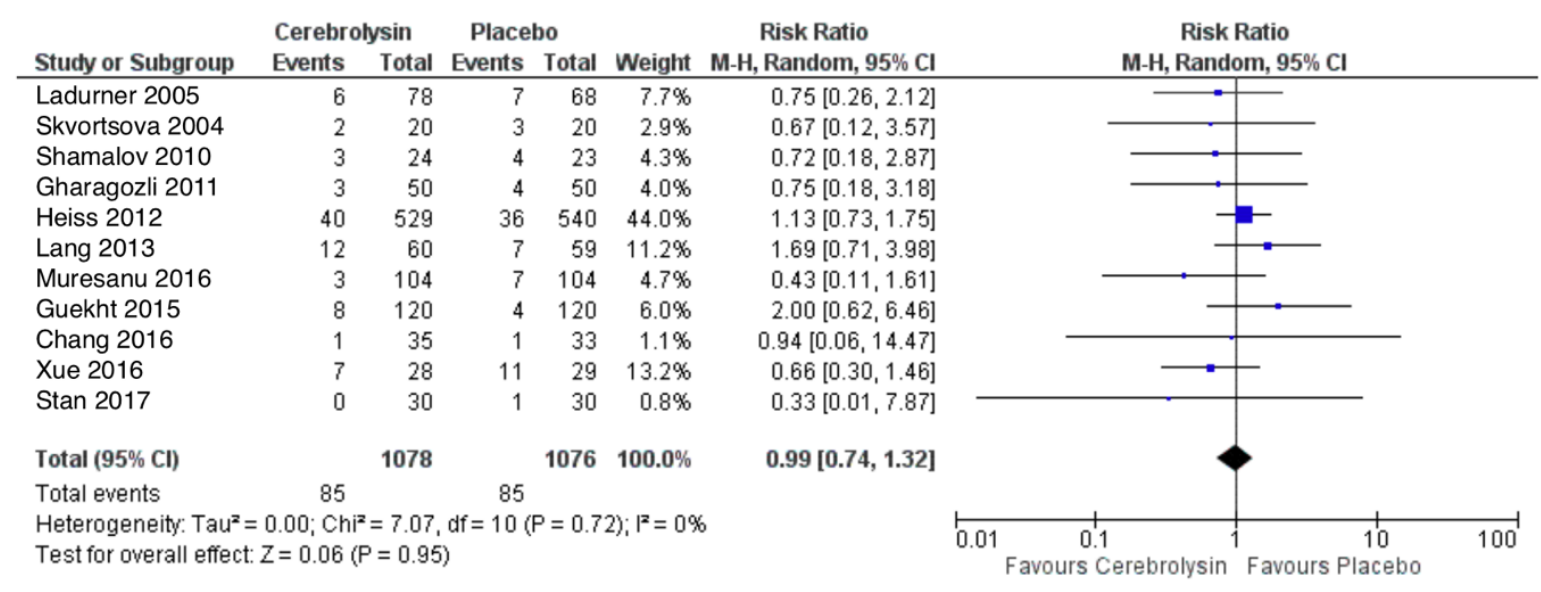

Figure 3. Serious adverse events (patients with at least one SAE), Comparison of Cerebrolysin versus Placebo in the Safety Population, Random Effects, M-H, Risk Ratio (RR)

\subsection{Adverse events (AE)}

$\mathrm{AE}$ were reported in a total of 472 out of 1078 subjects treated with Cerebrolysin (43.8\%), as compared to 470 out of 1078 subjects treated with placebo (43.6\%). The combined RR for patients with at least one $\mathrm{AE}$ showed no difference between the groups $(\mathrm{RR}=0.98,95 \% \mathrm{CI}=0.88$ to 1.09 , $P=0.73$, random effects model, Figure 4).

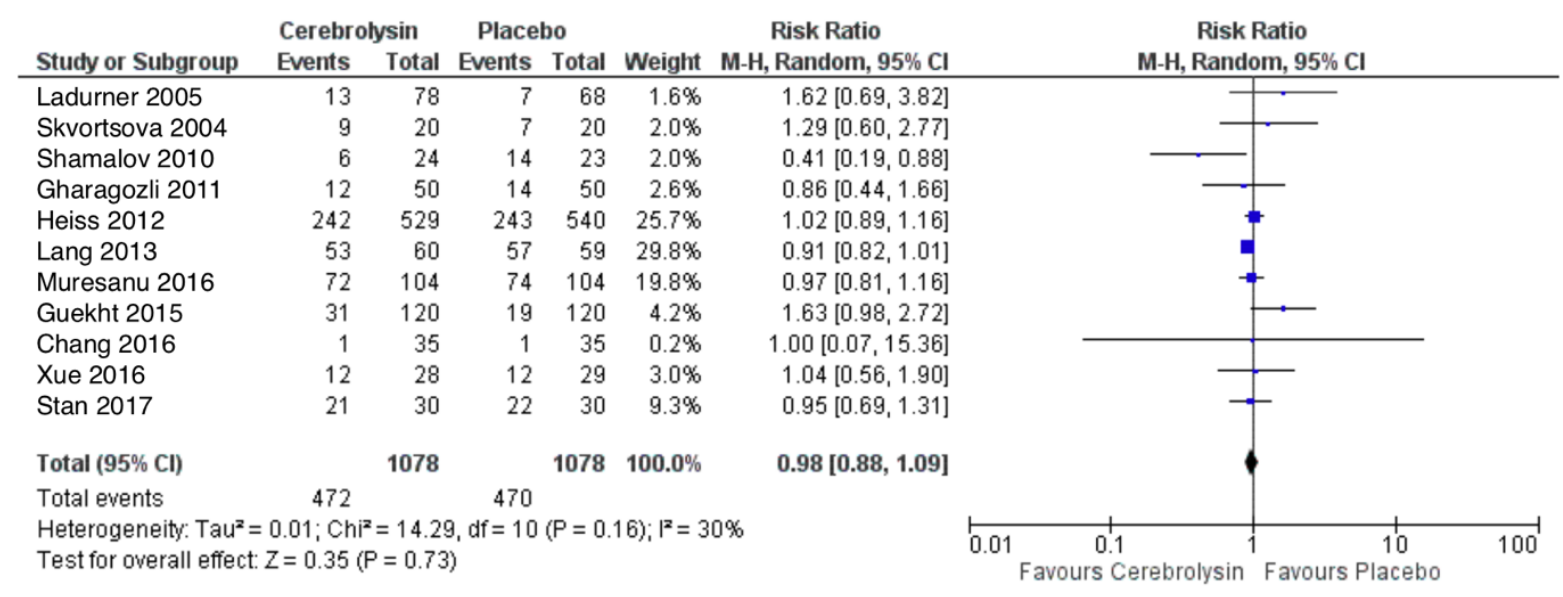

Figure 4. Adverse events (patients with at least one AE), Comparison of Cerebrolysin versus Placebo in the Safety Population, Random Effects, M-H, Risk Ratio (RR)

\subsection{Non-fatal serious adverse events (NF-SAE)}

NF-SAE were reported in a total of 41 out of 1078 subjects treated with Cerebrolysin (3.8\%), as compared to 32 out of 1078 subjects treated with placebo (3.0\%). The combined 
RR for patients with at least one NF-SAE showed a slightly higher rate in the Cerebrolysin group, which was statistically not significant with $\mathrm{P}=0.46(\mathrm{RR}=1.18,95 \% \mathrm{CI}=0.75$ to 1.86 , $\mathrm{P}=0.46$, random effects model, Figure 5).

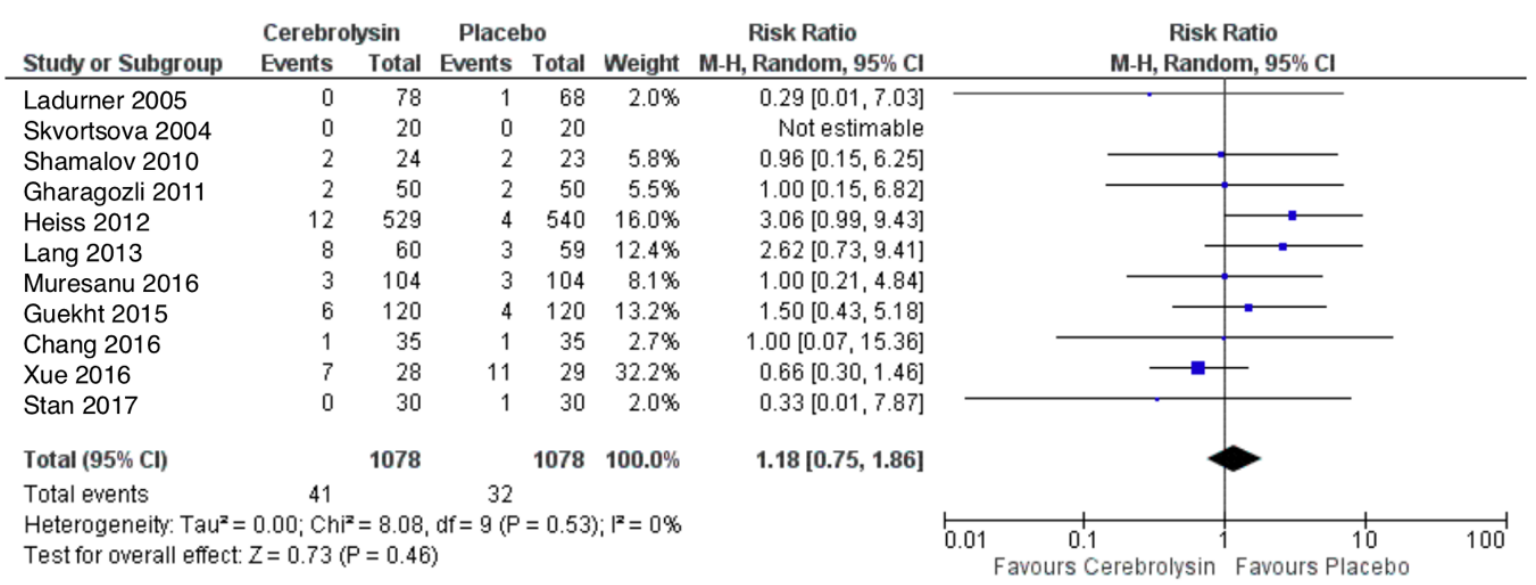

Figure 5. Non-fatal serious adverse events (patients with at least one NF-SAE), Comparison of Cerebrolysin versus Placebo in the Safety Population, Random Effects, M-H, Risk Ratio (RR)

\subsection{Sensitivity analyses}

All single subgroup results, as well as all formally combined subgroup results, were statistically not significant, well supporting the results of the crude pooling of all included randomized trials. Results from these analyses are present in Table 2. Effects for the 50ml subgroup treated for 20 days or more could not be estimated based on identified data.

Table 2. Results of subgroup sensitivity analyses. Effect estimates risk ratios are computed using the Mantel-Haenszel method

(M-H, random, 95\% Confidence Interval)

\begin{tabular}{|c|c|c|c|c|c|c|c|c|c|}
\hline \multirow{2}{*}{$\begin{array}{l}\text { Sample/ } \\
\text { Indicator }\end{array}$} & \multirow[t]{2}{*}{ All studies } & \multicolumn{3}{|c|}{ Cerebrolysin dose: $20-30 \mathrm{ml}$} & \multicolumn{2}{|c|}{ Cerebrolysin dose: $50 \mathrm{ml}$} & \multicolumn{2}{|l|}{ Initiation } & \multirow{2}{*}{$\begin{array}{l}\text { Studies available } \\
\text { online }\end{array}$} \\
\hline & & All & $<20$ days & $>=20$ days & All & $<20$ days & $<=24 \mathrm{~h}$ & $>24 h$ & \\
\hline \multicolumn{10}{|l|}{ Deaths } \\
\hline Sample size & 2202 & 1969 & 1351 & 518 & 233 & 233 & 1624 & 578 & 1962 \\
\hline \multirow[t]{2}{*}{ Effect estimate } & $0.83 \quad[0.57$ & $0.86[0.55$, & $0.88 \quad[0.56$ & $0.73 \quad[0.02$ & $0.75[0.32$, & $0.75[0.32,1.76]$ & $0.84 \quad[0.57$ & $0.73[0.02$, & $0.81[0.55,1.20]$ \\
\hline & $1.23]$ & $1.33]$ & 1.39] & $30.67]$ & $1.76]$ & & $1.25]$ & $30.67]$ & \\
\hline \multicolumn{10}{|l|}{ SAE } \\
\hline No. studies & 11 & 8 & 4 & 3 & 3 & 3 & 7 & 4 & 10 \\
\hline Sample size & 2154 & 1923 & 1305 & 518 & 233 & 233 & 1578 & 578 & 1914 \\
\hline
\end{tabular}




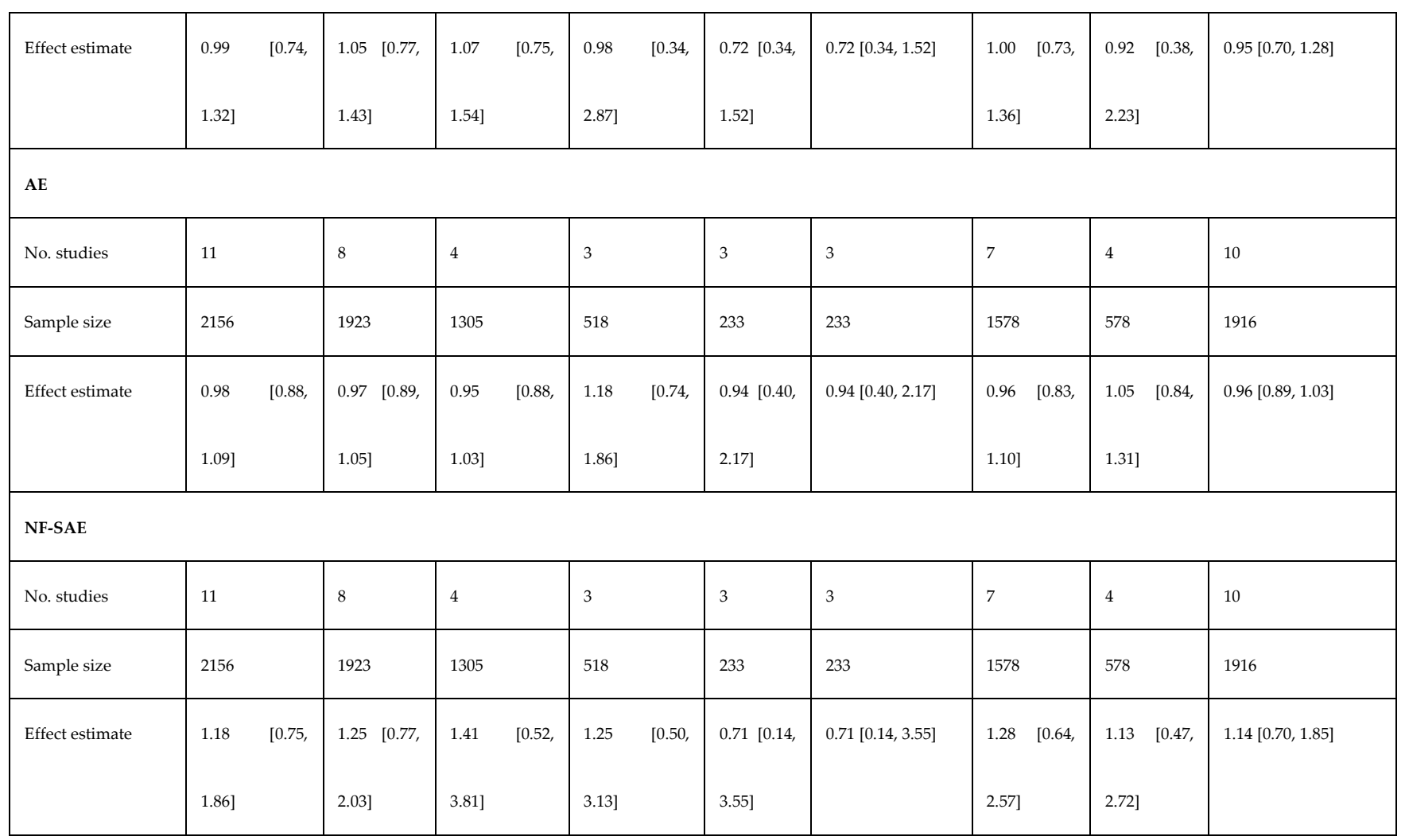

\section{Discussion}

To resolve the reported discrepancies between studies evaluating the safety of Cerebrolysin after acute ischemic stroke, the present meta-analysis aimed (1) to include a maximum number of RCTs and patients, and (2) to fill existing safety information gaps by following-up with primary source references and requesting supplementary material from original authors and producer of Cerebrolysin. Our pooled analysis of 2202 patients highlighted no indication for safety issues of Cerebrolysin. This was consistently observed throughout the pooled analyses of 12 RCTs, as well as throughout all subgroup analyses ( $p$-values $>0.05$ ). The least SAE rates as compared to placebo were found for the highest Cerebrolysin dose $(50 \mathrm{~mL})$, showing a moderate reduction of SAE as compared to placebo. Besides, there was a tendency for overall reduction of all-cause deaths. It is interesting to note that the least SAE and non-fatal SAE rates were found for the highest Cerebrolysin dose with $>25 \%$ risk reduction as compared to placebo.

The causes of SAE may be split into deaths and others, but these events are both SAE. Ziganshina et al. 2020 evaluated 6 studies for all-cause death (RR 0.9) [11]. However, for SAE and non-fatal SAE, they included only 4 studies. For the fatal SAE the above cited study included only three trials, even though information for fatal SAE was available in a total of six trials, and SAE was available for four studies. Gharagozli 2017 was evaluated for non-fatal SAE but not for fatal SAE (despite having 1 Cerebrolysin death and 2 placebo deaths) [12]. The reason for this approach may lie in the PICO of the review, namely that "all of the deaths occured within the seven-day acute-phase post-stroke period, owing to the severity of stroke". Gharagozli et al. writes in the article "three patients died in the acute phase due to stroke severity". For consistency, exclusion of such patients from fatal SAE analysis, usually warrants a similar approach for non-fatal SAE. We therefore assert that the Ziganshina 2020 et al. fatal vs non-fatal SAE evaluation faces two key limitations which both are not formally addressed in the review: (1) reduction of fatal SAE analysis to studies with non-fatal SAE information only, and (2) the exclusion of one trial from fatal 
SAE analysis without specifying a general rule to so or to describe the selection as a special subset from the total fatal SAE population.

In our safety-meta, we include 12 studies who provide details on SAE. For all studies, fatal and non-fatal SAE are explicitely reported as such (either in publication or CSR). Some trials had only few deaths but no other SAE (non-fatal $=0)$. One trial had no information on SAE: Amiri-Nikpour. This study is included in the meta-analysis on deaths, thus the meta-analysis on deaths includes 12 trials, the meta-analyses on SAE, non-fatal SAE etc. include consistenly 11 trials. The SAE definition is not related to the presumed cause of the event (e.g. "prolongation of existing hospitalization" is SAE also if directly related to the underlying disease. Additionally, the time of occurence plays no role for the classification as an SAE (except "within human drug trial").

As part of the limiting factors of this meta-analysis, there was a large heterogeneity of the trials with respect to baseline stroke severity: NIHSS trial medians were reaching from 7 to 14 . A stratitified analysis on studies with mild (NIHSS $<8$ ) versus moderatesevere (NIHSS $\geq 8$ ) stroke provided no indication for impact on safety results (all interaction $\mathrm{P} \geq 0.8$ ), with one exception: for mild vs. moderate-severe stroke the test for subgroup differences regarding patients with at least one $\mathrm{AE}$ indicated moderate heterogeneity (I2 $=63.6 \%, \mathrm{P}=0.10$ ), with lower risk ratios favouring Cerebrolysin in the moderate-severe subgroup (RR 0.95, $\mathrm{P}=0.33$ ), as compared to higher risk ratios in the mild subgroup (RR $1.26, \mathrm{P}=0.16)$. Another limitation is the restricted information on study conduct from some of the included trials despite special requests for provision of additional information. In one study there was no specific information available on serious or non-serious adverse events (Amiri-Nikpour 2014). Another limitation is the lack of prolonged observations (6 months, 1 year), which were not available from randomized clinical trials. These should be considered within the framework of future study designs.

A strength of the current paper is the inclusion of the largest number of studies on Cerebrolysin after stroke so far, comprising a total of 12 randomized double-blind trials. An important advantage is the inclusion of supplementary material, requested from the original authors if publications with summarized safety sections were not providing enough data for all safety outcomes of interest, a problem many such studies are confronted with. Therefore, a maximum of safety-related data could be obtained. Another strength is the homogentiy of the safety results across all sensitivity analyses, supporting the main result and demonstrating the robustness of the safety results across all analysis pathways.

This comprehensive safety meta-analysis shows a very good safety profile for patients treated with Cerebrolysin after acute ischemic stroke as compared to placebo. While none of the analyses provided evidence for safety issues, there was a tendency to superiority of Cerebrolysin regarding serious adverse events in high dose treatments and in moderate-severe stroke. Further randomized clinical trials are required to provide sufficient evidence also after discharge (day 90) and with longer, repetitive treatment cycles.

Supplementary Materials: The following are available online at www.mdpi.com/xxx/s1, Table S1: Risk of Bias across studies included in the safety meta-analysis.

Author Contributions: Conceptualization, S.S., L.V., D.B., A.P., P.R. and L.B.; methodology, S.S., D.B., A.P., L.B.; software, S.S.; validation, S.S., L.V., D.B., A.P., P.R. and L.B.; formal analysis, investigation, data curation, S.S., D.B. and A.P.; writing - original draft preparation, review and editing, S.S., L.V., D.B., A.P., P.R. and L.B.; visualization, S.S.; supervision, P.R, L.B.; All authors have read and agreed to the published version of the manuscript.

Funding: This research received no external funding.

Institutional Review Board Statement: Not applicable.

Informed Consent Statement: Not applicable. 


\begin{abstract}
Data Availability Statement: In this section, please provide details regarding where data supporting reported results can be found, including links to publicly archived datasets analyzed or generated during the study. Please refer to suggested Data Availability Statements in section "MDPI Research Data Policies" at https://www.mdpi.com/ethics. You might choose to exclude this statement if the study did not report any data.
\end{abstract}

Conflicts of Interest: The authors declare no conflict of interest.

\title{
References
}

1. Gorelick, P.B. The Global Burden of Stroke: Persistent and Disabling. The Lancet Neurology 2019, 18, 417-418, doi:10.1016/S1474-4422(19)30030-4.

2. Markus, H.S.; Brainin, M. COVID-19 and Stroke-A Global World Stroke Organization Perspective. Int J Stroke 2020, 15, 361-364, doi:10.1177/1747493020923472.

3. Gache, K.; Leleu, H.; Nitenberg, G.; Woimant, F.; Ferrua, M.; Minvielle, E. Main Barriers to Effective Implementation of Stroke Care Pathways in France: A Qualitative Study. BMC Health Services Research 2014, 14, 95, doi:10.1186/1472-696314-95.

4. Muresanu, D.F.; Strilciuc, S.; Stan, A. Current Drug Treatment of Acute Ischemic Stroke: Challenges and Opportunities. CNS Drugs 2019, doi:10.1007/s40263-019-00663-x.

5. Muresanu, D.F.; Heiss, W.-D.; Hoemberg, V.; Bajenaru, O.; Popescu, C.D.; Vester, J.C.; Rahlfs, V.W.; Doppler, E.; Meier, D.; Moessler, H.; et al. Cerebrolysin and Recovery After Stroke (CARS): A Randomized, Placebo-Controlled, Double-Blind, Multicenter Trial. Stroke 2016, 47, 151-159, doi:10.1161/STROKEAHA.115.009416.

6. Stan, A.; Birle, C.; Blesneag, A.; Iancu, M. Cerebrolysin and Early Neurorehabilitation in Patients with Acute Ischemic Stroke: A Prospective, Randomized, Placebo-Controlled Clinical Study. J Med Life 2017, 10, $216-222$.

7. Beghi, E.; Binder, H.; Birle, C.; Bornstein, N.; Diserens, K.; Groppa, S.; Homberg, V.; Lisnic, V.; Pugliatti, M.; Randall, G.; et al. European Academy of Neurology and European Federation of Neurorehabilitation Societies Guideline on Pharmacological Support in Early Motor Rehabilitation after Acute Ischaemic Stroke. European Journal of Neurology n/a, doi:10.1111/ene.14936.

8. Platz, T. Rehabilitative therapy for arm paresis following a stroke 2020.

9. Teasell, R.; Hussein, N.; Mirkowski, M.; Vanderlaan, D.; Saikaley, M.; Longval, M.; Iruthayarajah, J. Stroke Rehabilitation Clinician Handbook 2020.

10. Bornstein, N.M.; Guekht, A.; Vester, J.; Heiss, W.-D.; Gusev, E.; Hömberg, V.; Rahlfs, V.W.; Bajenaru, O.; Popescu, B.O.; Muresanu, D. Safety and Efficacy of Cerebrolysin in Early Post-Stroke Recovery: A Meta-Analysis of Nine Randomized Clinical Trials. Neurol. Sci. 2018, 39, 629-640, doi:10.1007/s10072-017-3214-0.

11. Ziganshina, L.E.; Abakumova, T.; Vernay, L. Cerebrolysin for Acute Ischaemic Stroke. Cochrane Database Syst Rev 2016, 12, CD007026, doi:10.1002/14651858.CD007026.pub4.

12. Gharagozli, K.; Harandi, A.A.; Houshmand, S.; Akbari, N.; Muresanu, D.F.; Vester, J.; Winter, S.; Moessler, H. Efficacy and Safety of Cerebrolysin Treatment in Early Recovery after Acute Ischemic Stroke: A Randomized, Placebo-Controlled, Double-Blinded, Multicenter Clinical Trial. J Med Life 2017, 10, 153-160.

13. Heiss, W.-D.; Brainin, M.; Bornstein, N.M.; Tuomilehto, J.; Hong, Z.; Cerebrolysin Acute Stroke Treatment in Asia (CASTA) Investigators Cerebrolysin in Patients with Acute Ischemic Stroke in Asia: Results of a Double-Blind, Placebo-Controlled Randomized Trial. Stroke 2012, 43, 630-636, doi:10.1161/STROKEAHA.111.628537.

14. Lang, W.; Stadler, C.H.; Poljakovic, Z.; Fleet, D.; Lyse Study Group A Prospective, Randomized, Placebo-Controlled, Double-Blind Trial about Safety and Efficacy of Combined Treatment with Alteplase (Rt-PA) and Cerebrolysin in Acute Ischaemic Hemispheric Stroke. Int J Stroke 2013, 8, 95-104, doi:10.1111/j.1747-4949.2012.00901.x.

15. Guekht, A.; Vester, J.; Heiss, W.-D.; Gusev, E.; Hoemberg, V.; Rahlfs, V.W.; Bajenaru, O.; Popescu, B.O.; Doppler, E.; Winter, S.; et al. Safety and Efficacy of Cerebrolysin in Motor Function Recovery after Stroke: A Meta-Analysis of the CARS Trials. Neurol Sci 2017, 38, 1761-1769, doi:10.1007/s10072-017-3037-z.

16. Ladurner, G.; Kalvach, P.; Moessler, H.; Cerebrolysin Study Group Neuroprotective Treatment with Cerebrolysin in Patients with Acute Stroke: A Randomised Controlled Trial. J Neural Transm (Vienna) 2005, 112, 415-428, doi:10.1007/s00702004-0248-2.

17. Skvortsova, V.I.; Stakhovskaia, L.V.; Gubskiǔ, L.V.; Shamalov, N.A.; Tikhonova, I.V.; Smychkov, A.S. [A randomized, double-blind, placebo-controlled study of Cerebrolysin safety and efficacy in the treatment of acute ischemic stroke]. Zh Nevrol Psikhiatr Im S S Korsakova 2004, 51-55.

18. Shamalov, N.A.; Stakhovskaia, L.V.; Burenchev, D.V.; Kichuk, I.V.; Tvorogova, T.V.; Botsina, A.I.; Smychkov, A.S.; Kerbikov, O.B.; Moessler, H.; Novak, P.; et al. [The effect of cerebrolysin in dosage $50 \mathrm{ml}$ on the volume of lesion in ischemic stroke]. Zh Nevrol Psikhiatr Im S S Korsakova 2010, 110, 34-37.

19. Amiri-Nikpour, M.R.; Nazarbaghi, S.; Ahmadi-Salmasi, B.; Mokari, T.; Tahamtan, U.; Rezaei, Y. Cerebrolysin Effects on Neurological Outcomes and Cerebral Blood Flow in Acute Ischemic Stroke. Neuropsychiatr Dis Treat 2014, 10, 2299-2306, doi:10.2147/NDT.S75304. 
20. Guekht, A.; Vester, J.; Heiss, W.-D.; Gusev, E.; Hoemberg, V.; Rahlfs, V.W.; Bajenaru, O.; Popescu, B.O.; Doppler, E.; Winter, S.; et al. Safety and Efficacy of Cerebrolysin in Motor Function Recovery after Stroke: A Meta-Analysis of the CARS Trials. Neurol Sci 2017, 38, 1761-1769, doi:10.1007/s10072-017-3037-z.

21. Chang, W.H.; Park, C.; Kim, D.Y.; Shin, Y.-I.; Ko, M.-H.; Lee, A.; Jang, S.Y.; Kim, Y.-H. Cerebrolysin Combined with Rehabilitation Promotes Motor Recovery in Patients with Severe Motor Impairment after Stroke. BMC Neurology 2016, 16, 31, doi:10.1186/s12883-016-0553-Z.

22. Xue, L.-X.; Zhang, T.; Zhao, Y.-W.; Geng, Z.; Chen, J.-J.; Chen, H. Efficacy and Safety Comparison of DL-3-n-Butylphthalide and Cerebrolysin: Effects on Neurological and Behavioral Outcomes in Acute Ischemic Stroke. Exp Ther Med 2016, 11, 2015-2020, doi:10.3892/etm.2016.3139. 